\title{
Probing the spatial dispersion in a dense atomic vapor near a dielectric interface
}

\author{
H. van Kampen, V. A. Sautenkov, ${ }^{*}$ E. R. Eliel, and J. P. Woerdman \\ Huygens Laboratory, Leiden University, P.O. Box 9504, 2300 RA Leiden, The Netherlands
}

(Received 17 November 1997)

\begin{abstract}
We have experimentally investigated the selective reflection of the $P-D$ transition in a high-density rubidium vapor and shown it to be sensitive to the exact spatial distribution of $P$-state atoms near the dielectricvapor interface. This distribution results from the radiative and nonradiative transport of excitation and from wall-quenching collisions of excited-state atoms and is highly inhomogeneous. Selective reflection thus acts as a probe of the spatial dispersion in the vapor. The resulting spectra have been analyzed to show that the broadening due to $S-D$ collisions contributes considerably to the self-broadened linewidth of the $P-D$ transition. [S1050-2947(98)07712-9]

PACS number(s): 32.70.Jz, 42.50.-p
\end{abstract}

\section{INTRODUCTION}

A medium is said to exhibit spatial dispersion when its dielectric coefficient $\epsilon$ not only depends on the frequency $\omega$ of the incident electromagnetic wave but also on the wave vector $\vec{k}$ thereof: $\boldsymbol{\epsilon}=\boldsymbol{\epsilon}(\omega, \vec{k})$. Usually, spatial dispersion arises in media that are inhomogeneous, i.e., where $\epsilon$ depends on the spatial coordinates. Well-known examples of these media are layered materials [1]. The optical properties of such media strongly depend on the (mis)match between layer thickness and wavelength of the incident radiation and only weakly on the variation of the dielectric coefficients of the layer materials with frequency [1].

Spatial dispersion also comes into play in the optical response of an atomic vapor in the vicinity of dielectric interfaces. If the vapor is dilute the spatial dispersion arises from the effect that the atomic polarization gets quenched when an atom hits the dielectric and responds transiently to the incident laser field when the atom moves away from the dielectric again. The atomic polarization is restored to its steadystate value after a time of the order of the natural lifetime. This effect can be described in terms of a nonlocal dielectric coefficient and leads to sub-Doppler structures in the selective-reflection spectrum [2-4].

In addition, spatial dispersion arises in a dense atomic vapor as a consequence of nonuniformity of linear and nonlinear absorption processes. As an example, it has been predicted that a self-reflected wave is created at the sharp boundary of saturated and unsaturated regions inside a dense vapor [5]. Obviously, the optical response of the vapor is considerably different at either side of this boundary and the medium is thus spatially dispersive. Note that here the spatial dispersion results from a self-consistent description of the system of atoms and light field: the position and sharpness of the boundary depend on both the intensity of the incident light field and the atomic density. Apart from being caused by spatially dependent saturation effects, spatial dispersion can also arise in a dense vapor when the incident optical field

\footnotetext{
*Present address: Physics Department, Texas A\&M University, College Station, TX 77843-4242.
}

does not saturate the medium. All that is required is a nonzero excited-state population that is spatially inhomogeneous on the scale of the optical wavelength.

In this paper we study spatial dispersion in a dense atomic vapor due to inhomogeneous excitation near the window of a vapor cell. Here the excitation profile is created by using laser light tuned near the center of the fundamental resonance transition of the atoms. At sufficiently high vapor densities such light is absorbed over a length $l_{a}^{0}=\lambda / 2 \pi$, with $\lambda$ the resonance wavelength, resulting in an excitation rate that decays on the same length scale. The spatial distribution of excited atoms in such a vapor, however, does not simply follow the spatial variation of the excitation rate. It is modified by radiative [6] and nonradiative transport processes inside the vapor and excitation quenching at the window-vapor interface [7-9]. The spatial distribution of excited atoms resulting from the interplay of these processes is inhomogeneous on the scale of an optical wavelength and so will be the atomic polarization. As a consequence, spatial dispersion arises in the vapor. In our experiment we directly probe its influence on the spectral response of the vapor.

Since the spatial dispersion originates in the spatial density distribution of the excited atoms $n(z)$, our probe has to be sensitive to $n(z)$ itself rather than to its spatially integrated value; the latter is the case for absorption or fluorescence spectroscopy. In contrast, selective-reflection spectroscopy probes the atomic polarization, and thus $n(z)$, within an optical wavelength distance from the interface [10]. Since in our experiment the excited-state density distribution is inhomogeneous over a wavelength we use selective-reflection spectroscopy as a probe for $n(z)$. We consider a transition from the excited state (e.g., an alkali-metal-atom $P$ state) to a higher-lying state (e.g., an alkali-metal-atom $D$ state) rather than a transition from a ground state (e.g., an alkali-metalatom $S$ state) to a first-excited state since the former is more sensitive to the $P$-state density $n(z)$. In this way we study the effect of the spatial inhomogeneity of the distribution of $P$-state atoms and demonstrate that the resulting spatial dispersion has major consequences for the line shape of the $P-D$ transition.

The study of the spectral response of the $P-D$ transition in reflection not only gives information about the spatial dispersion in the vapor but also on the collisional broadening of 


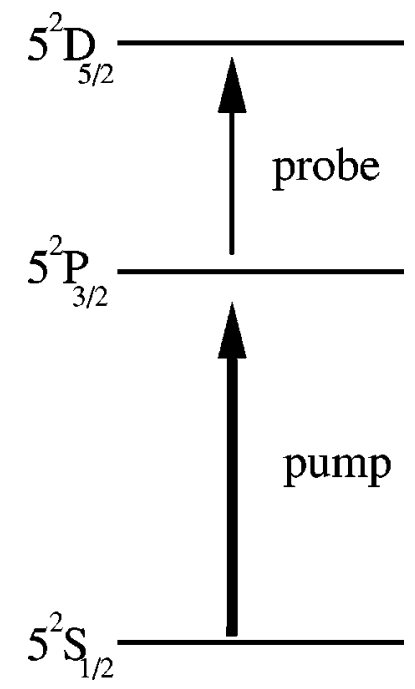

FIG. 1. Cascade three-level system of rubidium.

the spectral line involved. In this way we can obtain information on the atom-atom interaction. It has been argued that the broadening of the $P-D$ transition is effectively equal to that of the fundamental $S-P$ transition. This is because the van der Waals interaction and the quadrupole-quadrupole interaction between a $D$-state atom and a ground $S$-state atom have a considerably shorter range as compared to the resonant dipole-dipole interaction between $S$ - and $P$-state atoms [11]. The comparison of the $S-P$ and the $P-D$ spectral widths opens the way to test this statement.

In this paper we present results of a two-step excitation experiment. We perform a selective-reflection study of both the fundamental $S-P$ and the $P-D$ transitions in a high density rubidium vapor $\left[N=(0.2-2.2) \times 10^{17} \mathrm{~cm}^{-3}\right]$. In Sec. II we describe the experimental technique, in Sec. III we give a theoretical model for the reflectivity including a nonuniform distribution of $P$-state atoms, in Sec. IV we present the results and discussion, and in Sec. V we give the conclusions.

\section{EXPERIMENTAL TECHNIQUE}

In our experiment we excite rubidium atoms from the $5^{2} S_{1 / 2}$ ground state to the $5{ }^{2} P_{3 / 2}$ excited state $\left(D_{2}\right.$ line $)$. This transition at $\lambda_{S-P}=780 \mathrm{~nm}$ (Fig. 1) is driven by a Ti:sapphire laser using a power of $5 \mathrm{~mW}$ in a $1 \mathrm{~mm}$ diameter beam. The intensity $\left(<1 \mathrm{~W} / \mathrm{cm}^{2}\right)$ is such that the transition is not saturated at the relevant $\mathrm{Rb}$ densities; the resulting fraction of atoms in the $5^{2} P_{3 / 2}$ state is estimated to be less than $1 \%$ [12]. Coherent effects, such as a shift or splitting of the levels involved, are negligible [13]. An external-cavity semiconductor laser (New Focus model 6224), operating at $\lambda_{P-D}=775.8 \mathrm{~nm}$, is used to probe the $5^{2} P_{3 / 2}-5^{2} D_{3 / 2}$ transition in reflection. For the study of the linewidth of the fundamental $S-P$ transition, as discussed in the Introduction, the probe laser is tuned to $780 \mathrm{~nm}$. In all cases the intensity of the probe light is kept to a very low value $(0.1 \mathrm{~mW})$ in order to avoid saturation.

The rubidium vapor is contained in the rear section of an all-sapphire cell (see Fig. 2) that is heated to produce high atomic densities. The vapor density is determined by the temperature of the coldest spot of the cell and is varied be-

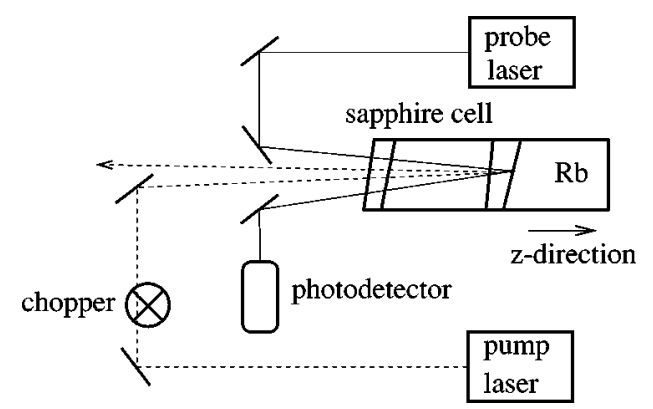

FIG. 2. Experimental pump-probe setup to measure the selective-reflection spectrum on both the $S-P$ and $P-D$ transitions in a high density rubidium vapor.

tween $0.2 \times 10^{17}$ and $2.2 \times 10^{17} \mathrm{~cm}^{-3}$. Except at the lowest densities, the self-broadened full width at half maximum $\Gamma_{\text {self }}$ of the fundamental transition exceeds the combined broadening due to hyperfine structure, Doppler effect, and isotopic composition: $\Gamma_{\text {self }} / N=2 \pi \times 1 \times 10^{-7} \mathrm{~cm}^{3} \mathrm{~s}^{-1}$ [14]. The front and rear sections of the cell are separated by a wedged sapphire window; the front section is evacuated to avoid turbulence and thus fluctuations in the position of the optical beams. The incoming pump and probe beams propagate almost collinearly and are overlapped at the cell window at near-normal angles of incidence. The pump-laser beam is amplitude modulated with a chopper at $2 \mathrm{kHz}$ and the probe beam, reflected from the window-vapor interface, is detected with a combination of a photodiode and a lock-in amplifier.

\section{THEORETICAL MODEL}

The theory for the reflectivity of an interface between a dielectric and an atomic vapor in the vicinity of the fundamental resonance transition of the atoms, i.e., selective reflection, has been discussed extensively $[3,10,15]$. We will restrict the discussion to the case at hand: a high-density vapor, for which only collisional broadening has to be taken into account, probed on a $P-D$ transition with spatial dispersion. The dispersion results from a spatially inhomogeneous density distribution of $P$-state atoms, $n=n(z)$; the $z$ axis is oriented perpendicular to and has its origin at the dielectric. A plane wave with complex field amplitude $E_{0}$ and wave vector $k$, parallel to the interface normal, propagates in the dielectric medium towards the interface and is partially reflected back into the dielectric. The field that is refracted into the atomic vapor induces a spatially dependent dipole polarization that radiates back into the dielectric. This radiated field adds coherently to the field that is reflected back by the bare dielectric interface. The total complex reflected-field amplitude $E$ can therefore be written as

$$
E=E_{v}+E_{p},
$$

with $E_{v}$ the reflected-field amplitude in the absence of the vapor, i.e., for vacuum and $E_{p}$ the contribution to the reflected field due to the dipole polarization induced in the gas-phase atoms by the field in the vapor. The field amplitude $E_{p}$ can generally be written as

$$
E_{p}=\frac{i k}{(\eta+1) \epsilon_{0}} \int_{0}^{\infty} d z \exp (i k z) P_{0}(z)
$$


Here $k=k_{0} \eta$ is the magnitude of the wave vector inside the dielectric, $\eta$ the refractive index of the dielectric, $\epsilon_{0}$ the dielectric constant in vacuum, and $P_{0}(z)$ the $z$-dependent dipole polarization in the vapor.

The fact that we probe the $P-D$ transition allows us to considerably simplify the theoretical model. This is because the $P-D$ oscillator strength is roughly one order of magnitude smaller as compared to that of the fundamental resonance transition while their linewidths are of the same order. This implies that the dielectric coefficient for frequencies around the $P-D$ resonance frequency is, for all densities, very close to unity. In the vicinity of the fundamental resonance frequency, however, $\epsilon(\omega)$ can differ considerably from one for sufficiently high densities. In that case the dipole polarization in the vapor has to be calculated using a selfconsistent model [15]. Because $\epsilon(\omega) \approx 1$ for the $P-D$ transition we can neglect local-field effects [16] and take the field in the vapor simply proportional to the incident field. We can then evaluate the interface reflectivity to first order in the polarization and find for the reflection coefficient $R$ $=\left|E / E_{0}\right|^{2}$ the expression [10]

$$
R=\left|\frac{\eta-1}{\eta+1}\right|^{2}+\frac{4 \eta(\eta-1)}{(\eta+1)^{3}} \operatorname{Re} T,
$$

where $T$ is determined by the dipole polarization $P_{0}(z)$. In order to find an expression for $T$ [Eq. (3)] we solve the optical Bloch equations for the $P-D$ transition in the limit that the population density of the $D$ state is negligible. The result is

$$
T=\frac{i k|\mu|^{2}}{2 \epsilon_{0} \hbar}\left(\frac{-\Delta_{p}+i \Gamma}{\Delta_{p}^{2}+\Gamma^{2}}\right) \int_{0}^{\infty} d z n(z) \exp (2 i k z),
$$

with $\mu$ the $P$ - $D$ transition dipole moment, $\Delta_{p}$ the detuning from resonance of the probe laser and $\Gamma$ the collisional linewidth of the $P-D$ transition. Note that $T=0$ if the $S-P$ pump laser is absent $[n(z)=0]$. As can be seen from Eqs. (3) and (4) the exact spatial distribution $n(z)$ of the population in the $P$ state directly enters into the interface reflectivity. The integral in Eq. (4) can be written as a $c$ number,

$$
\int_{0}^{\infty} d z n(z) \exp (2 i k z)=A+i B,
$$

leading to

$$
T=\frac{-k|\mu|^{2}}{2 \epsilon_{0} \hbar}\left[\frac{A \Gamma-B \Delta_{p}+i\left(B \Gamma+A \Delta_{p}\right)}{\Delta_{p}^{2}+\Gamma^{2}}\right] .
$$

In our experiment the laser that transfers atoms to the $P$ state is amplitude modulated. Our lock-in signal is therefore proportional to the difference in reflectivity $\Delta R$ for the case that the $P$ state is populated and the case that it is not populated,

$$
\Delta R=\frac{4 \eta(\eta-1)}{(\eta+1)^{3}} \frac{k|\mu|^{2}}{2 \epsilon_{0} \hbar} \frac{A \Gamma-B \Delta_{p}}{\Delta_{p}^{2}+\Gamma^{2}} .
$$

To illustrate our claim that the frequency-dependent reflectivity modulation strongly depends on the distribution of excited atoms we take a naive model where we assume that the

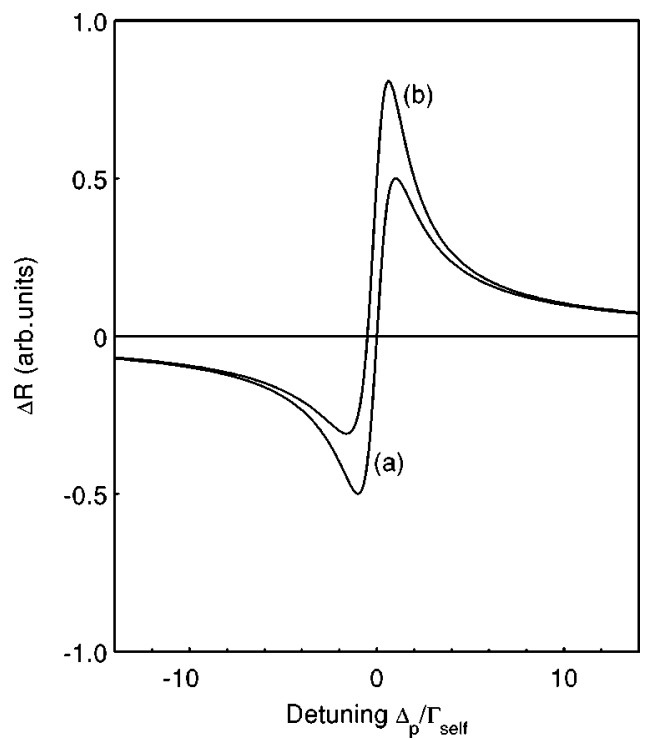

FIG. 3. Qualitative plot of the frequency dependence of the atomic contribution $\Delta R$ to the interface reflectivity on the probe transition for situations where the excited-state population is spatially homogeneous [curve (a)] and where the excited-state population decays exponentially over a length of $\lambda_{S-P} / 2 \pi$ [curve (b)]. Note that for the latter case the zero crossing of $\Delta R$ does not correspond to the center of the line.

excited-state density $n(z)$ is proportional to the local intensity $I(z)=I_{0} \exp (-\alpha z)$ with $\alpha$ the absorption coefficient of the pump light. In the case that $\alpha$ is small the population in the $P$ state is spatially uniform on the scale of a wavelength, i.e., $n(z)=n_{0}$, and one finds $A=0$ and $B=n_{0} / 2 k$. The spectrum of $\Delta R$ is then a purely dispersion-shaped Lorentzian [see Fig. 3 curve (a)]. When $\alpha$ is large the pump light is strongly absorbed over a distance $\alpha^{-1}=\lambda_{S-P} / 2 \pi$ and the density distribution is $n(z)=n_{0} \exp \left(-2 \pi z / \lambda_{S-P}\right)$. We then find for the $A$ and $B$ coefficients in Eq. (7) the values $A$ $=n_{0} \alpha /\left(\alpha^{2}+4 k^{2}\right) \quad$ and $B=2 n_{0} k /\left(\alpha^{2}+4 k^{2}\right) \quad$ with $k$ $=2 \pi / \lambda_{P-D}$. The reflectivity $\Delta R$ now contains dispersive (odd in detuning) and absorptive (even in detuning) contributions with a ratio $B / A=2\left(\lambda_{S-P} / \lambda_{P-D}\right)$ as shown in Fig. 3, curve (b). Note that our assumptions regarding the excitedstate density distribution are too naive; a more realistic picture of this distribution involves quenching of the atomic excitation at the vapor-window interface. This picture will be discussed in the following section.

\section{RESULTS AND DISCUSSION}

We have studied the selective-reflection spectra of rubidium on the $P-D$ transition for densities $N=(0.2-2.2)$ $\times 10^{17} \mathrm{~cm}^{-3}$. Except for the lowest densities the collisional width of the $S-P$ transition is, as discussed above, larger than the hyperfine and isotope splittings and Doppler width of the transition [17]; at sufficiently high densities this results in a single broad spectral line. For the $P-D$ transition this is true for all densities since the excited states have much smaller hyperfine splittings as compared to that of the ground state. Typical results for $N=0.6 \times 10^{17} \mathrm{~cm}^{-3}$ are shown in Fig. 4 . Here we show the $P-D$ spectra for three values of the detuning $\Delta$ of the pump laser [18]. The dashed curves are the 


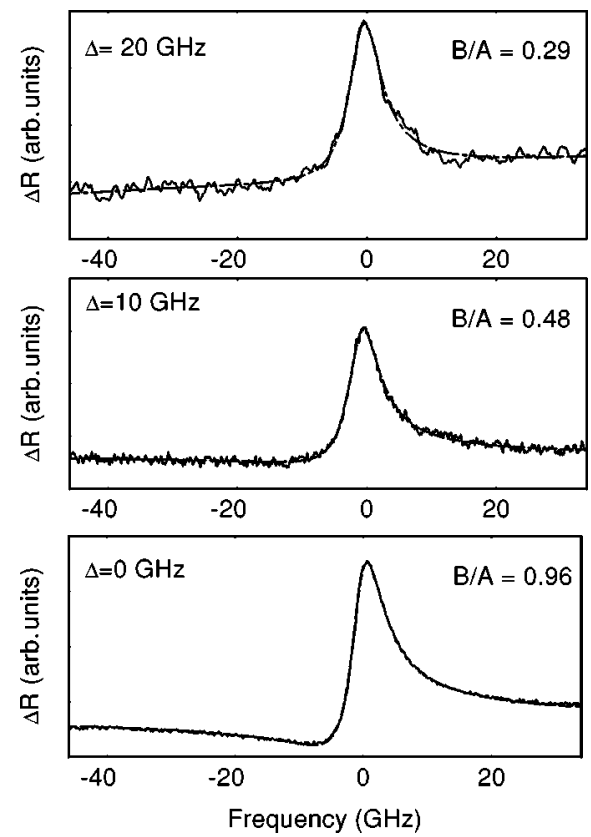

FIG. 4. Experimental selective-reflection spectra on the $P-D$ transition in a high density rubidium vapor $\left(N=0.6 \times 10^{17} \mathrm{~cm}^{-3}\right)$ for three different detunings $\Delta$ of the pump laser. The dashed curves show fitted Lorentzians that have both dispersive and absorptive components. The ratios of the weight factors of the dispersive $(B)$ and absorptive $(A)$ parts are shown in each frame.

results of a fit through the data of a sum of a dispersive and absorptive Lorentzian line shape function with equal widths [Eq. (7)]. Figure 4 clearly shows that the shape of the $P-D$ selective-reflection spectrum is sensitive to the detuning of the pump laser. In all cases the curves have both a dispersive and an absorptive component, the former becoming less and less important for increasing values of the pump detuning. This clearly contradicts the prediction at the end of Sec. III. In the naive model that was used there the process of quenching at the vapor-window interface was not included. The experimental results indicate that that approximation is not justified.

We attribute the spectral changes to modifications of the nonuniform distribution of $P$-state atoms as a function of pump detuning. Note that the changes in the $P-D$ line shape can be observed because a separate pump laser is used to create a $P$-state distribution, and thus a volume polarization, that is nonuniform. For the $S-P$ transition using a single laser it has been shown that a nonuniform polarization is created due to an exponentially attenuating field in the vapor at sufficiently high densities; obviously the attenuation factor depends on the detuning of the laser relative to the fundamental resonance frequency. In this case the nonuniformity of the polarization gives rise to a shift of the spectral line but leaves its shape fundamentally unaffected [15].

The cause of the nonuniformity in a dense atomic vapor near a dielectric interface is well understood: Excited-state atoms that collide with the dielectric become quenched. This process gives rise to a negligible excited-state density at the position of the interface, $n(0)=0$. Furthermore, the excitedstate density at small $z$ is determined by a combination of nonradiative diffusive transport of excitation, mediated by resonant dipole-dipole collisions between ground- and

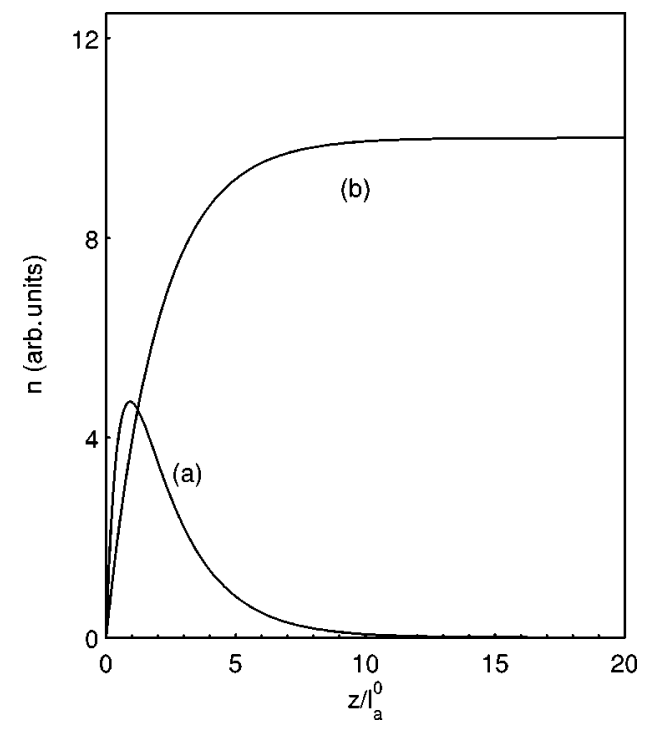

FIG. 5. Schematic spatial distribution of excited atoms, the $\mathrm{z}$ axis being calibrated in units of line-center absorption length $l_{a}^{0}$. Curve (a) shows the density distribution of excited atoms when the pump laser is tuned to resonance $(\Delta=0)$ and curve (b) shows the distribution of excited atoms for the case in which the pump laser is tuned far into the wing of the $S$ - $P$ transition.

excited-state atoms, and the local excitation rate [7-9]. For small values of $z$ one can write for the excited-state density distribution [7]

$$
n(z)=\frac{S_{0}}{D\left(l_{a}^{-2}-l_{\tau}^{-2}\right)}\left[e^{-z / l_{\tau}-e^{-z / l_{a}}}\right],
$$

valid for the case that $l_{a} \leqslant l_{\tau}$ as will be discussed below. Here $l_{a}$ is the absorption length for the pump light, $S_{0}$ the excitation rate per unit volume at the dielectric interface, and $l_{\tau}$ $=\sqrt{D \tau}$ the distance over which the excitation diffuses within the atomic lifetime $\tau$ with $D \propto N^{-1}$ the excitation-diffusion coefficient. Interestingly, we have an experimental handle on the excited-state distribution of Eq. (8) through the absorption length $l_{a}$. At fixed atomic density one can write $l_{a}$ $=l_{a}^{0}\left[\left(2 \Delta / \Gamma_{\text {self }}\right)^{2}+1\right]$ with $l_{a}^{0}=\lambda_{S-P} / 2 \pi$ the line-center absorption length at sufficiently high densities, $\Gamma_{\text {self }}$ the full width at half maximum of the self-broadened resonance line, and $\Delta$ the detuning of the pump laser. The excited-stated distribution can thus be modified through the detuning of the pump laser.

The line-center absorption length $l_{a}^{0}$ of a dense $\mathrm{Rb}$ vapor excited at its fundamental resonance equals $124 \mathrm{~nm}$. For a vapor with density $N=0.6 \times 10^{17} \mathrm{~cm}^{-3}$ the diffusion length $l_{\tau}=483 \mathrm{~nm}\left(D=0.083 \mathrm{~cm}^{2} \mathrm{~s}^{-1}\right.$ and $\left.\tau=28 \mathrm{~ns}\right)$. The inequality $l_{a}^{0}<l_{\tau}$ thus applies for all densities relevant to our experiment. The excited-state density distribution therefore follows Eq. (8) when the pump laser is tuned to the center of the $S-P$ transition $(\Delta=0)$. At sufficiently large values of the pumplaser detuning $\Delta$ one enters the regime where $l_{a} \gg l_{\tau}$. In this regime radiation trapping is important and the excited-state density distribution is no longer described by Eq. (8) [7]. Figure 5 shows the distribution $n(z)$ for the case that $\Delta=0$ so that $l_{a}=l_{a}^{0}$ [curve (a)]; it also shows [curve (b)] a qualitative solution for $n(z)$ for the situation where $l_{a} \gg l_{\tau}$. In the 
latter limit the distribution of excited-state atoms is homogeneous except for a region near the interface where $n(z)$ falls to zero over a distance characterized by the diffusion length $l_{\tau}$. Because the reflection spectrum is sensitive to the spatial distribution of excited atoms it is an obvious conclusion that the reflection spectrum will be substantially different in the two cases discussed above and we essentially probe the spatial dispersion near the dielectric-vapor interface.

We fit our experimental spectra with a combination of dispersive and absorptive Lorentzians of equal width [Eq. (7)]; the results are included in Fig. 4 (dashed curves). The ratio of the weight factors $A$ and $B$ is also shown in Fig. 4 for each of the displayed curves. We see that our model provides an excellent description of the line shape of the $P-D$ transition. The values of $B / A$ that we extract from our experiments are in good agreement with our model description if we use Eq. (8) and solve the integral in Eq. (5). For instance, when the pump laser is tuned near resonance $(\Delta \approx 0)$ the spatial distribution of excited atoms is highly inhomogeneous, as shown in Fig. 5 [curve (a)]. For this case, we calculate $B / A=0.96$ in perfect agreement with the experimental result $(B / A=0.96)$. When the pump laser is tuned away from resonance the distribution of excited atoms is much more smooth as shown in Fig. 5 [curve (b)]. A calculation of the line shape based on Eq. (8) shows that the ratio $B / A$ becomes smaller with increasing value of $\Delta$ : for $\Delta=10 \mathrm{GHz}$ and $\Delta$ $=20 \mathrm{GHz}$ we find $B / A=0.34$ and $B / A=0.25$, respectively, in reasonable agreement with the experimental results $(B / A$ $=0.48$ and $B / A=0.29$, respectively). Note that at this density $\Gamma_{\text {self }}=6 \mathrm{GHz}$. The reason for the discrepancy between experiment and theory for excitation in the line wing $(\Delta$ $\left.>\Gamma_{\text {self }}\right)$ is that in this case the spatial distribution of excited atoms is not accurately described by Eq. (8).

Because we have such an excellent description of the line shape of the $P-D$ transition (see Fig. 4) we can extract its self-broadened width $\Gamma=\Gamma_{\text {self }}^{P-D}$ as a function of density. In the same setup we can measure the full width at half maximum $\Gamma_{\text {self }}^{S-P}$ of the $S-P$ transition by tuning the probe laser to the fundamental transition and switching off the pump laser. The latter width is deduced from the difference $\Gamma_{\mathrm{SR}}$ between the frequency of maximum reflectivity and that of minimum reflectivity in the reflection spectrum [19]. For a Lorentzian spectral line in the case of a sapphire-rubidium vapor interface one can show that for a $D_{2}$ line $\Gamma_{\mathrm{SR}}=1.33 \Gamma_{\text {self }}^{S-P}$ using the fact that the refractive index of sapphire is 1.76. Both $\Gamma_{\text {self }}^{S-P}(\square)$ and $\Gamma_{\text {self }}^{P-D}(\triangle)$ are linear functions of density as shown in Fig. 6. A least-squares fit through the data for the two self-broadened transitions yields values for the selfbroadening coefficients: $\quad k_{S-P}=\Gamma_{\text {self }}^{S-P} / N=2 \pi \times 0.8$ $\times 10^{-7} \mathrm{~s}^{-1} \mathrm{~cm}^{3}$, in good agreement with literature values [14] and $k_{P-D}=\Gamma_{\text {self }}^{P-D} / N=2 \pi \times 1 \times 10^{-7} \mathrm{~s}^{-1} \mathrm{~cm}^{3}$. We estimate the experimental error to be 5\%. This result shows that the $P-D$ line is more strongly broadened than the fundamental resonance transition. This can be understood as follows: the fundamental transition is dominantly broadened as a result of resonance-exchange collisions between atoms in the ground state and atoms in the excited $P$ state. The interatomic potential for these $S$ - $P$ collisions has a $R^{-3}$ dependence where $R$ is the interatomic distance. The interatomic potential for collisions between two ground-state atoms is

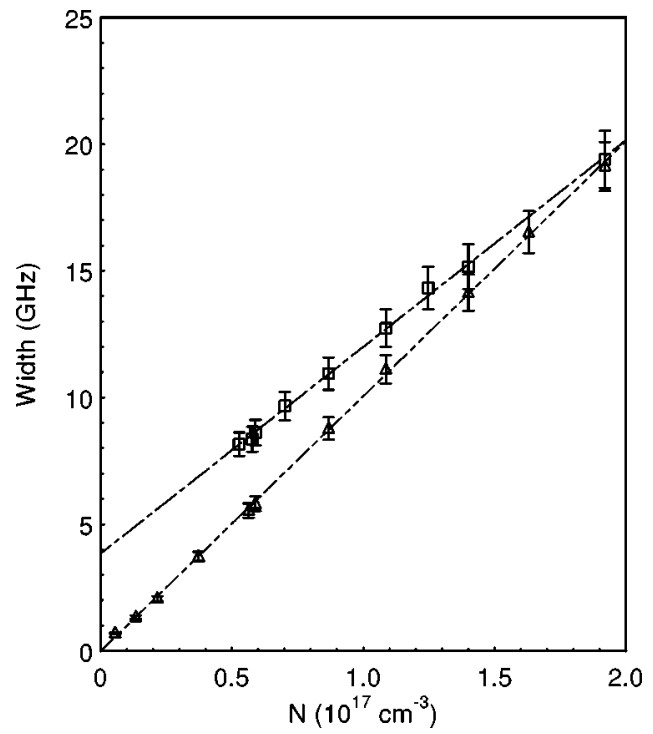

FIG. 6. The full self-broadened linewidth at half maximum $\Gamma_{\text {self }}^{S-P}$ of the fundamental $S-P(\square)$ transition and of the $P-D(\triangle)$ transition $\left(\Gamma_{\text {self }}^{P-D}\right)$ as obtained in our two-step selective-reflection experiment as a function of the vapor density $N$. The dashed lines indicate the results of linear fits.

van der Waals-like with a $R^{-6}$ potential. This potential has a much shorter range; therefore it does not play an important role in a description of the $S$ - $P$ linewidth. The last relevant potential is the $S-D$ potential where both the van der Waals interaction $\left(R^{-6}\right)$ and the resonant quadrupole-quadrupole interaction $\left(R^{-5}\right)$ take part [20]. The linewidth of the $P-D$ transition is then determined by both the resonance broadening of the fundamental transition and the broadening due to $S$ - $D$ collisions. Our experimental results allow one to estimate the contribution of the $S$ - $D$ collisions to the linewidth of the $P-D$ transition as $\approx 20 \%$. This value is appreciably larger than the value of 5\% obtained in a two-photon experiment [20] in $\mathrm{Na}$, in line with the larger polarizability of $\mathrm{Rb}$ as compared to that of $\mathrm{Na}$; both the van der Waals and quadrupole-quadrupole contributions to the $S-D$ collisions become larger with increasing atomic number [21].

\section{CONCLUDING REMARKS}

In conclusion, we have experimentally shown that for a dense $\mathrm{Rb}$ vapor $\left(N=0.2-2.2 \times 10^{17} \mathrm{~cm}^{-3}\right)$ the spectral response of the $P-D$ transition in reflection is very sensitive to the exact spatial distribution of $P$-state atoms in the vicinity of the vapor-window interface. This distribution is inhomogeneous as a result of radiative and nonradiative transport of excitation and wall-quenching collisions of $P$-state atoms. The detailed $P$-state distribution is modified by the detuning of the excitation light relative to the $S$ - $P$ resonance line giving us a handle on the spatial dispersion in the vapor.

Furthermore, we have shown that a comparison of the $S$ - $P$ and $P$ - $D$ self-broadening coefficients yields information on the interaction between $S$ - and $D$-state atoms. Specifically, we have shown that $S$-D collisions are quite effective: they contribute $20 \%$ to the $P-D$ broadening coefficient. 


\section{ACKNOWLEDGMENTS}

We gratefully acknowledge C. J. C. Smeets and J. C. Visser for help with the experiments. This work is part of the research program of the "Stichting voor Fundamenteel Onderzoek der Materie', and was made possible by the financial support from the "Nederlandse Organisatie voor Wetenschappelijk Onderzoek.',
[1] P. Yeh, Optical Waves in Layered Materials (Wiley, New York, 1988).

[2] J. P. Woerdman and M. F. H. Schuurmans, Opt. Commun. 14, 248 (1975).

[3] M. F. H. Schuurmans, J. Phys. (France) 37, 469 (1976).

[4] F. Schuller, O. Gorceix, and M. Ducloy, Phys. Rev. A 47, 519 (1993).

[5] L. Roso-Franco, Phys. Rev. Lett. 55, 2149 (1985); J. Opt. Soc. Am. B 4, 1878 (1987).

[6] T. Holstein, Phys. Rev. 83, 1159 (1951).

[7] H. van Kampen, V. A. Sautenkov, A. M. Shalagin, E. R. Eliel, and J. P. Woerdman, Phys. Rev. A 56, 3569 (1997).

[8] A. G. Zajonc and A. V. Phelps, Phys. Rev. A 23, 2479 (1981).

[9] A. V. Phelps and A. O. McCoubrey, Phys. Rev. 118, 1561 (1960)

[10] G. Nienhuis, F. Schuller, and M. Ducloy, Phys. Rev. A 38, 5197 (1988); G. Nienhuis and F. Schuller, ibid. 50, 1586 (1994); F. Schuller, G. Nienhuis, and M. Ducloy, ibid. 43, 443 (1991).

[11] Z. J. Jabbour, J. Sagle, R. K. Namiotka, and J. Huennekens, J. Quant. Spectrosc. Radiat. Transf. 54, 767 (1995).

[12] J. J. Maki, W. V. Davis, R. W. Boyd, and J. E. Sipe, Phys. Rev. A 46, 7155 (1992).
[13] Y. R. Shen, The Principles of Nonlinear Optics (Wiley and Sons, New York, 1984), Chap. 22.

[14] K. Niemax and G. Pichler, J. Phys. B 8, 179 (1975); K. Niemax, M. Movre, and G. Pichler, ibid. 12, 3503 (1979).

[15] J. Guo, J. Cooper, A. Gallagher, and M. Lewenstein, Opt. Commun. 131, 732 (1994); J. Guo, J. Cooper, and A. Gallagher, Phys. Rev. A 53, 1130 (1996); P. Wang, A. Gallagher, and J. Cooper, ibid. 56, 1598 (1997).

[16] M. Born and E. Wolf, Principles of Optics, 6th ed. (Pergamon, Oxford, 1980), p. 98.

[17] E. L. Lewis, Phys. Rep. 58, 1 (1980).

[18] The detuning $\Delta$ is determined relative to the atomic resonance frequency $\omega_{0}$ as extracted from a low-density absorption spectrum.

[19] The $S$ - $P$ selective-reflection spectrum is, in general, not a purely dispersive curve although this reflectivity is proportional to the spatial distribution of ground-state atoms, which is uniform. The distortion of the line shape results from the large number of ground-state atoms; therefore a linear expansion of the reflectivity in density is not allowed.

[20] J. P. Woerdman, Opt. Commun. 28, 69 (1979).

[21] K. Niemax, J. Quant. Spectrosc. Radiat. Transf. 17, 125 (1977); 17, 747 (1977). 